\title{
Synergistic induction of apoptosis in HeLa cells by the proteasome inhibitor bortezomib and histone deacetylase inhibitor SAHA
}

\author{
YIZHOU JIANG, YI WANG, ZIJIE SU, LING YANG, WEIWEI GUO, WEN LIU and JI ZUO \\ Department of Cellular and Genetic Medicine, Shanghai Medical College, Fudan University, Shanghai 200032, P.R. China
}

Received February 12, 2010; Accepted April 26, 2010

DOI: $10.3892 / \mathrm{mmr} 00000305$

\begin{abstract}
Proteasome inhibitors and histone deacetylase (HDAC) inhibitors are two promising groups of anti-cancer agents. In this study, we examined the apoptotic effects of the proteasome inhibitor bortezomib and HDAC inhibitor suberoylanilide hydroxamic acid (SAHA) in human cervical carcinoma HeLa cells. Compared to treatment with bortezomib or SAHA alone, co-exposure with these two agents synergistically resulted in the massive apoptosis of HeLa cells, consistent with a significant increase in caspase-3 activation. We then investigated the mechanisms underlying this effect. The combination of bortezomib and SAHA caused an increase in the ratio of bax/bcl-2 expression, inhibited the nuclear transportation of $\mathrm{NF}-\kappa \mathrm{B}$, and down-regulated Akt expression and phosphorylation in HeLa cells. In conclusion, bortezomib and SAHA cooperatively stimulate apoptosis in HeLa cells through the inhibition of several cyto-protective signalling pathways. This is the first report of synergistic apoptotic effect achieved with a proteasome inhibitor and HDAC inhibitor in HeLa cells. Thus, the results build the framework for clinical trials using combined proteasome and HDAC inhibition in the treatment of human cervical carcinoma.
\end{abstract}

\section{Introduction}

Human cervical carcinoma is one of the most common malignancies in women. Although conventional chemotherapy induces a high rate of complete remission, relapse is frequently observed and these patients have a poor clinical outcome (1).

Correspondence to: Dr Ji Zuo or Dr Wen Liu, Department of Cellular and Genetic Medicine, Shanghai Medical College, Fudan University, Shanghai 200032, P.R. China

E-mail: jzuo@shmu.edu.cn

Abbreviations: HDAC, histone deacetylase; BTZ, bortezomib; SAHA, suberoylanilide hydroxamic acid; GAPDH, glyceraldehyde phosphate dehydrogenase, MTT, 3-[4,5-dimethylthiazol-2-yl]-2,5diphenyltetrazolium bromide

Key words: proteasome inhibitor, histone deacetylase inhibitor, HeLa cells, apoptosis
Insights into the pathogenesis of human cervical carcinoma reveal alterations in cell proliferation and apoptosis, and the dysregulation of multiple intracellular signaling pathways, such as nuclear factor NF- $\mathrm{KB}$ and Akt (2). Therefore, improved approaches to treating cervical carcinoma are likely to result from the investigation of those non-chemotherapeutic agents that specifically target the pathways that cancer cells depend on for their survival.

Protein degradation by proteasomes is vital to cell viability, ensuring the exact regulation of the breakdown of the metabolic enzymes, transcription factors and proteins involved in the cell cycle and cell death. Proteasome inhibitors represent a new treatment strategy targeting the $26 \mathrm{~S}$ proteasome, a component of the ubiquitin-proteasome complex responsible for the degradation of unwanted cellular proteins. These inhibitors show significant activity against various cancer cells, leading to the induction of cellular apoptosis (3). Bortezomib (Velcade, PS-341) is the first clinically available proteasome inhibitor, and its use was recently reported in the treatment of human cervical carcinoma (4).

Mutation or aberrant expression of genes, especially those responsible for the cell cycle and apoptosis, is commonly related to the tumorigenesis of many types of cancer. The acetylation and deacetylation of histone play an important role in the regulation of these genes. Histone deacetylase (HDAC) inhibitors constitute a group of compounds that promote histone acetylation, chromatin uncoiling and the transcription of genes involved in multiple cellular processes, including apoptosis (5). Suberoylanilide hydroxamic acid (SAHA), a hydroxamic acid HDAC inhibitor, has shown single-agent efficacy in HeLa cells (6).

Previous studies have shown that the synchronous inhibition of the proteasome and HDAC functions against many non-solid tumors, including multiple myelomas, mantle cell lymphoma and T-cell lymphoma (7-9). However, the possible effects and therapeutic mechanisms of this combination in HeLa cells remain to be investigated. To address this issue, we examined the combined action of the proteasome inhibitor bortezomib with SAHA in HeLa cells in vitro. These two agents interact in a highly synergistic manner to induce apoptosis in HeLa cells. HeLa cells are associated with multiple perturbations in the apoptotic and survival pathways. Therefore, these results warrant further investigation of bortezomib and SAHA in combination as a potential therapy for human cervical carcinoma. 


\section{Materials and methods}

Cells and reagents. Human cervical carcinoma HeLa cells were grown in Dulbecco's modified Eagle's medium supplemented with $10 \%$ newborn bovine serum in a $5 \% \mathrm{CO}_{2}$ humidified atmosphere at $37^{\circ} \mathrm{C}$.

Bortezomib was obtained commercially (Millennium Pharmaceuticals, Cambridge, MA, USA) and SAHA (Aton Pharma, Tarrytown, NY, USA) was kindly provided by Merck \& Co., Inc. (Whitehouse Station, NJ, USA).

Primary antibodies against caspase-3, bax, bcl-2, NF- $\mathrm{kB}$, Akt and phosphorylated Akt (p-Akt) were obtained from Cell-Signaling Technology (Beverly, MA, USA). Antibodies against glyceraldehyde phosphate dehydrogenase (GAPDH) and Lamin-B1 were purchased from Abcam (Cambridge, UK). Horseradish peroxidase-conjugated goat anti-mouse IgG and goat anti-rabbit IgG were obtained from Cell Signaling Technology.

3-[4,5-dimethylthiazol-2-yl]-2,5-diphenyltetrazoliumbromide (MTT) reduction assay. The determination of cell viability was carried out using the MTT reduction assay, based on the reduction of tetrazolium salt. In brief, MTT (Pufei, Shanghai, P.R. China) was added to the culture medium at the end of incubation. After incubation with MTT at $37^{\circ} \mathrm{C}$ for $4 \mathrm{~h}$, the media was removed and the cells were lysed and formazandissolved with $150 \mu \mathrm{l}$ dimethyl sulfoxide. Absorbance was measured at $492 \mathrm{~nm}$ with a microplate reader (Thermo Scientific, Anaheim, CA, USA), and results were expressed as the percentage of MTT reduction, assuming the absorbance of control cells as $100 \%$.

Determination of synergistic effect. Synergistic interaction is determined by improved Loewe analysis as described previously (10). Briefly, the $\mathrm{IC}_{50}$ values of bortezomib and SAHA were defined as the concentration needed for a $50 \%$ reduction in absorbance calculated based on the cell viability curve using the MTT assay, HeLa cells were treated with mixtures of bortezomib and SAHA, and the appropriate volumes were used to obtain drug mixtures at seven ratios (0:5, 1:4, 2:3, 1:1, 3:2, 4:1 and 5:0) at their predicted $\mathrm{IC}_{50}$ values (e.g., a 1:1 ratio was prepared by mixing an equal volume of bortezomib at its predicted $\mathrm{IC}_{50}$ with SAHA at its predicated $\mathrm{IC}_{50}$ ). The combination index (CI) was defined as $C I=d_{1} / D y_{1}+d_{2} / D y_{2}$, where $d_{1}$ and $d_{2}$ are the doses of bortezomib and SAHA in mixture, respectively. This produces an effect $y ; D y_{1}$ and $D y_{2}$ were the doses that produced the same effect as $y$ when used alone. The combination dose $\left(d_{1}\right.$, $d_{2}$ ) was determined to be synergistic when the CI was $<1$.

\section{Assessment of apoptosis}

Giemsa and Hoechst 33258 staining. Cell morphology was examined using Giemsa and Hoechst 33258 staining. In brief, cells were seeded on poly-L-lysine-coated coverslips for $24 \mathrm{~h}$, treated with the drugs for the indicated times and then fixed with $4 \%$ paraformaldehyde. The coverslips were stained with Giemsa or Hoechst 33258. Five hundred cells from each slide were examined and counted under a fluorescence microscope (Leica, Wetzlar, Germany).

Electron microscopy. Electron microscopy was used to examine the ultra-structure of the HeLa cells. Cells were rinsed in $0.1 \mathrm{M}$ phosphate buffer ( $\mathrm{pH}$ 7.4), post-fixed for $1 \mathrm{~h}$ in $1 \%$ osmium tetroxide, dehydrated in alcohol and embedded in epoxy resin. Semi-thin sections (1- $\mu \mathrm{m}$ thick) were stained with $1 \%$ toluidine blue to locate areas of interest for electron microscopic examination. Ultrathin sections stained with uranyl acetate and lead citrate were examined under a TEM-100CX electron microscope (Japan Electron Optical Laboratory, Tokyo, Japan).

Real-time quantitative RT-PCR. Total RNA was isolated from HeLa cells after treatment for the indicated times using TRIzol reagent (Invitrogen) according to the manufacturer's instructions. The first-strand cDNA was synthesized with a Revert Aid first strand cDNA synthesis kit (MBI Fermentas, Vilnius, Lithuania). cDNA, primers and iQSYBR Green SuperMix (Bio-Rad, Hercules, CA, USA) in a final volume of $25 \mu 1$ were used for PCR. Amplification and detection of specific products were carried out in a Mini opticon real-time PCR system (Bio-Rad) under the following conditions: 40 cycles at $94^{\circ} \mathrm{C}$ for $30 \mathrm{sec}, 58^{\circ} \mathrm{C}$ for $30 \mathrm{sec}$ and $72^{\circ} \mathrm{C}$ for $40 \mathrm{sec}$. Specific primers were used to amplify cDNA for bcl-2 (5'-TCCGATCAGAAGGCTAGAGTT-3'; 5'-TCGGTCTCC TAAAAGCAGGC-3') and for bax (5'-TCCCCCCGA GAGGTCTTTT-3'; 5'-CGGCCCCAGTTGAAGTTG-3'). As the internal control, 18S RNA primers (5'-ATCCCT GAAAAGTTCCAGCA-3'; 5'-CCCTCTTGGTGAGGTCA ATG-3') were used for RNA template normalization.

Whole cell protein extraction and Western blot analysis. Cells were lysed in RIPA buffer [150 mM NaCl, $1 \%$ NP-40, $0.5 \%$ Doc, $0.1 \%$ sodium dodecyl sulfate and $50 \mathrm{mM}$ Tris/HCl (pH 8.0)] supplemented with $1 \mu \mathrm{g} / \mathrm{ml}$ aprotinin and $100 \mu \mathrm{g} /$ $\mathrm{ml}$ phenylmethylsulfonyl fluoride. The cell suspension was incubated on ice for $30 \mathrm{~min}$ then centrifuged at $20,000 \mathrm{x} \mathrm{g}$ for $15 \mathrm{~min}$ at $4^{\circ} \mathrm{C}$. The supernatants were collected for further analysis. The protein concentration of the samples was determined by the Bradford assay. A total of $20 \mu \mathrm{g}$ of proteins was separated by 10 or $15 \%$ sodium dodecyl sulfatepolyacrylamide gels and transferred onto nitrocellulose membranes (Amersham Pharmacia Biotech, Little Chalfont, UK). The membranes were blocked with $5 \%(\mathrm{~W} / \mathrm{V})$ fat-free dry milk in TBS-T buffer ( $20 \mathrm{mM}$ Tris-HCl, $\mathrm{pH} 7.6,137 \mathrm{mM}$ $\mathrm{NaCl}$ and $0.05 \%$ Tween-20) and incubated overnight at $4{ }^{\circ} \mathrm{C}$ with the relevant primary antibodies. Blocked membranes were washed and incubated with the appropriate horseradish peroxidase-conjugated secondary antibodies for $45 \mathrm{~min}$. Immunocomplexes were visualized using the enhanced chemiluminescence Western blotting detection system (CellSignaling Technology) with exposure of the membranes to X-ray film (Eastman Kodak, Rochester, NY, USA). GAPDH and Lamin B1 were used to ensure equivalent loading of whole and nuclear cellular protein, respectively. The signal intensities of the respective bands were quantified by a scanning densitometer using an image analysis system with Scion Image version 4.03 software.

Statistical analysis. Data were expressed as the mean \pm SEM of at least three independent experiments. Statistical significance was determined using ANOVA. $\mathrm{P}<0.05$ was considered significant. 


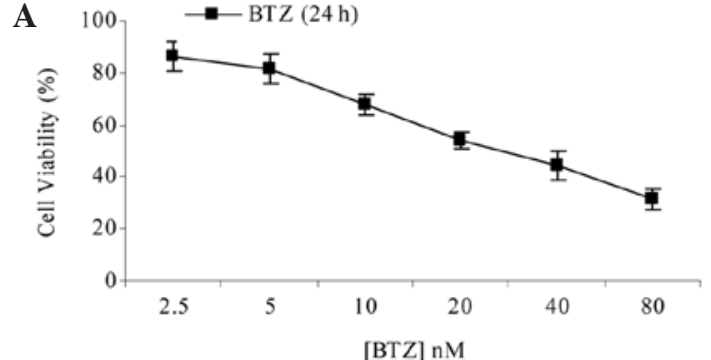

C

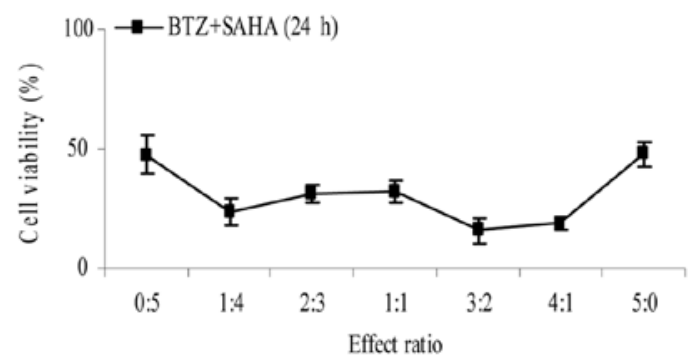

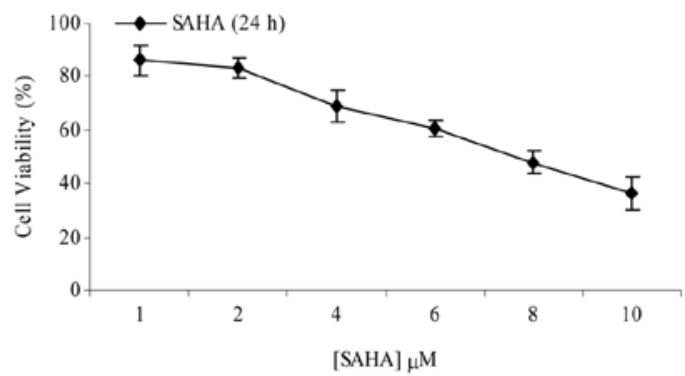

D

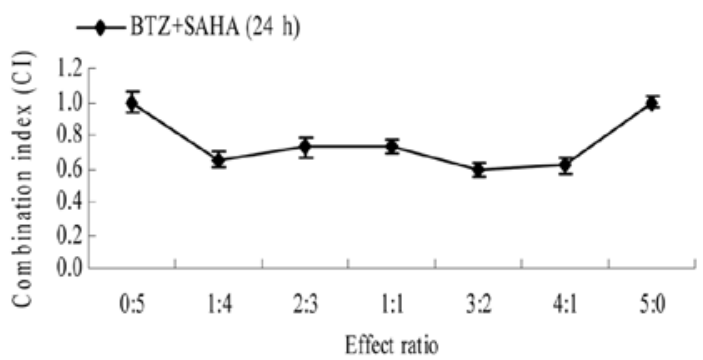

Figure 1. Decrease in viability of HeLa cells caused by the combination of bortezomib and SAHA. Survival rate of HeLa cells after treatment with bortezomib (A) and SAHA (B). Cell viability was analyzed by the MTT assay. (C) Exposure of HeLa cells to various effect ratios of the combination of bortezomib and SAHA. Significant growth inhibition was observed as compared to bortezomib or SAHA treatment alone with a maximum synergistic effect at an effect ratio of 3:2 (BTZ:SAHA). Data were obtained from at least three experiments. (D) The combination index (CI) of co-treatment at various effect ratios of bortezomib and SAHA.

\section{Results}

Bortezomib and SAHA in combination induced synergistic cytotoxicity in HeLa cells. To determine the combined effect of bortezomib and SAHA on cell viability, we first examined the survival rate of HeLa cells after treatment with bortezomib and SAHA. The MTT assay showed that both bortezomib (2.5-80 $\mathrm{nM})$ (Fig. 1A) and SAHA (1-10 $\mu \mathrm{M})$ (Fig. 1B) treatment markedly reduced HeLa cell viability in a dose-dependent manner after $24 \mathrm{~h}$. At a fixed anticipated inhibition rate of $50 \%\left(\mathrm{IC}_{50}\right)$, HeLa cells were treated for $24 \mathrm{~h}$ with drug combinations of different ratios. Compared to each agent alone, a significant decrease in the cell survival rate was observed with the combined treatment (Fig. 1C). Median doseeffect analysis yielded a CI value of $<1.0$ for the combination at all different ratios, indicating a synergistic interaction between bortezomib and SAHA (Fig. 1D). For example, $5.6 \mathrm{nM}$ bortezomib and $4.3 \mu \mathrm{M}$ SAHA alone induced a reduction in cell viability of $\sim 20$ and $30 \%$, respectively; however, in combination they achieved a $>80 \%$ reduction (Fig. 1D). Treatment with the combination of drugs was used in the following studies to achieve the maximal inhibitory effect. These data suggest that bortezomib and SAHA synergistically decrease the viability of HeLa cells.

Bortezomib and SAHA in combination synergistically triggered apoptosis in HeLa cells. To determine whether the growth inhibition induced by bortezomib and SAHA in HeLa cells was caused by apoptosis, we employed Giemsa and Hochest 33258 staining to examine the morphological changes in HeLa cells after treatment. After treatment with bortezomib or SAHA alone for $24 \mathrm{~h}, \sim 50 \%$ of the HeLa cells showed the characteristic morphological changes of apoptosis, including shrinking of the cytoplasm and nuclear fragmentation with an intact cell membrane (Fig. 2A-a) or a contracted nucleus and condensed chromatin fragments (Fig. 2A-b). The amount of apoptotic cells was dramatically increased after co-treatment with bortezomib and SAHA, with $>80 \%$ of HeLa cells determined as apoptotic. The apoptotic cells were quantified and it was revealed that co-treatment resulted in a significant increase in apoptosis compared to single treatment $(\mathrm{P}<0.05$, Fig. 2B).

After co-treatment with bortezomib and SAHA, cell morphology was further analyzed by electron microscopy. A considerable number of cells presented the features of late apoptosis, including cell shrinkage, nuclear condensation and fragmentation, and the formation of apoptotic bodies (Fig. 2C). By contrast, the cells treated with bortezomib or SAHA alone only showed some signs of early apoptosis. Chromatin condensation only occurred at the peripheral areas of the nuclei, and few apoptotic bodies were observed. These data suggest that the combination of bortezomib and SAHA expedites the process of apoptosis in HeLa cells.

To further confirm the level of apoptosis in HeLa cells after co-treatment with bortezomib and SAHA, Western blot analysis was used to examine the cleavage of caspase- 3 in cells under various treatments. As showed in Fig. 2D, bortezomib in combination with SAHA significantly induced the cleavage of caspase-3, whereas single treatment exerted minimal effects (Fig. 2D). No cleavage of caspase-3 was detected in untreated cells. These data demonstrate that bortezomib in conjunction with SAHA represents a potent stimulus for caspase-3 activation and apoptosis in HeLa cells.

Bortezomib and SAHA in combination enhanced the expression ratio of bax/bcl-2. To further investigate the mechanism 

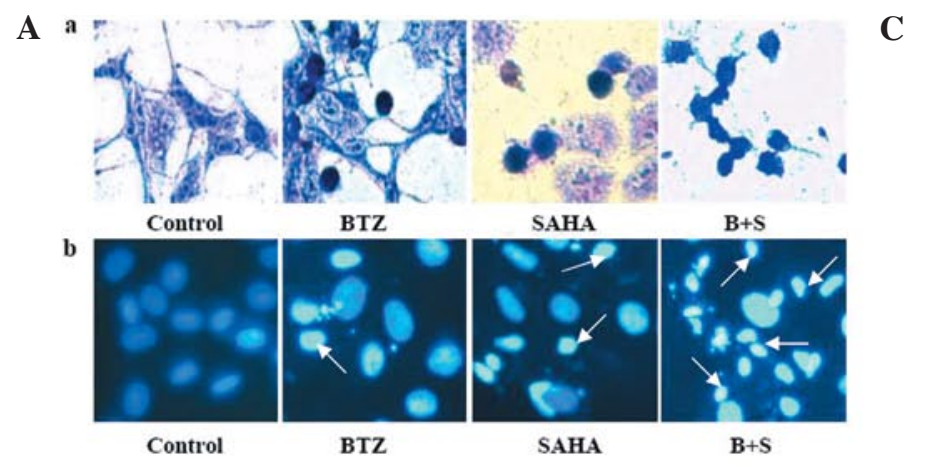

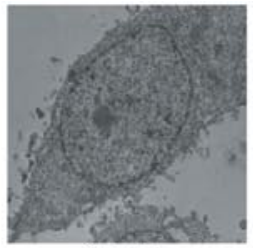

Control

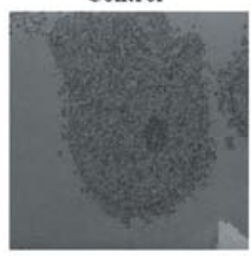

SAHA

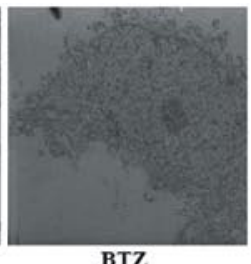

BTZ

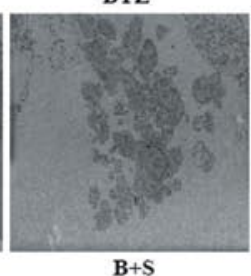

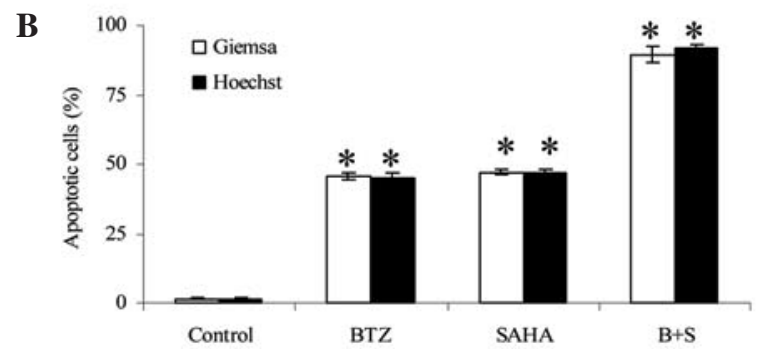

D

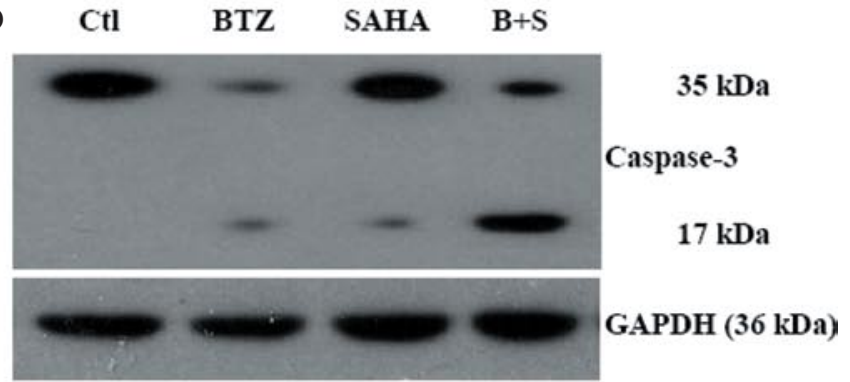

Figure 2. Induction of apoptosis in HeLa cells by the combination of bortezomib and SAHA. (A-a) Giemsa staining of HeLa cells after the indicated treatments. (A-b) Hoechst 33258 staining showing the nuclei of HeLa cells. Arrows indicate the nuclei of apoptotic cells (magnification x200). (B) Apoptotic cell numbers expressed as a percentage of total cells. ${ }^{*} \mathrm{P}<0.05$ vs. control. Data were obtained from at least three experiments. (C) Morphological features of apoptotic cells (after co-treatment with $5.6 \mathrm{nM}$ bortezomib and $4.3 \mu \mathrm{M} \mathrm{SAHA}$ ) examined by electron microscopy. (D) Western blot analysis of the cleavage of caspase-3 after the co-treatment.

underlying the corporative effect of bortezomib and SAHA in apoptosis induction in HeLa cells, we decided to examine whether bortezomib and SAHA treatment affect the ratio of pro-apoptotic bax and anti-apoptotic bcl-2 at both the transcription and protein levels. Real-time RT-PCR results revealed an increase in bax mRNA expression and a decrease in bcl-2 mRNA expression, which led to a substantial increase in the bax/bcl-2 ratio (Fig. 3A). Compared to the control group, there was no significant change in bax or bcl-2 mRNA expression in single-treated cells. Western blotting was then used to analyze the expression of bax and bcl-2 proteins after the co-treatment. The level of bax protein was increased after co-treatment, whereas less bcl-2 protein was detected in the HeLa cells (Fig. 3B and C). Consistent with the gene expression, the protein ratio of bax/bcl-2 was highly increased after co-treatment of bortezomib and SAHA. There was no change in bax or bcl-2 protein expression between the single-treated and control groups.

This indicates that the combination of bortezomib and SAHA induces massive apoptosis by altering the gene and protein expression of bcl-2 and bax.

Bortezomib and SAHA in combination affected $N F-\kappa B$ nuclear translocation and Akt expression. The NF- $\mathrm{KB}$ pathway is a key pathway that promotes the expression of proliferative and anti-apoptotic genes. It has been demonstrated that the functions of NF- $\mathrm{KB}$ depend on its cellular distribution (11). To determine whether NF- $\kappa B$ is involved in the synergistic apoptotic effect of bortezomib and SAHA, we examined the alteration in NF- $\mathrm{KB}$ distribution in the HeLa cells. Compared to the control, bortezomib and SAHA alone had little effect on cytoplasmic NF- $\mathrm{KB}$ expression, though nuclear NF- $\mathrm{KB}$ expression was slightly enhanced (Fig. 4A). Despite the elevated level of NF- $\mathrm{KB}$ observed in the cytoplasm of the HeLa cells, after the co-treatment there was a significant decrease in the amount of nuclear NF- $\mathrm{KB}$ (Fig. 4A and B). These data indicate that the combination of bortezomib and SAHA affects the nuclear translocation of NF- $\kappa B$ in HeLa cells, suggesting that the dysfunction of NF- $\kappa \mathrm{B}$ may be involved in the cooperative apoptotic effect of bortezomib and SAHA.

In addition, the Akt pathway has important functions in cell survival and proliferation and is frequently hyperactivated in most human cancers. It regulates many downstream signal molecules, including NF- $\kappa \mathrm{B}, \mathrm{Bcl}-2$ family proteins and murine double minute 2 , via phosphorylation and activation of key molecules (12). Therefore, we finally examined the expression and phosphorylation of Akt in HeLa cells. There was no notable change in Akt protein expression in HeLa cells after treatment with bortezomib or SAHA alone. The phosphorylation of Akt was decreased in HeLa cells after bortezomib treatment, but not after SAHA treatment. More importantly, co-treatment with bortezomib and SAHA induced a marked reduction in the level of Akt and its phophorylation. These data imply that Akt pathway may also be associated with the enhanced apoptosis induced by the combination of bortezomib and SAHA (Fig. 4C and D). 
A
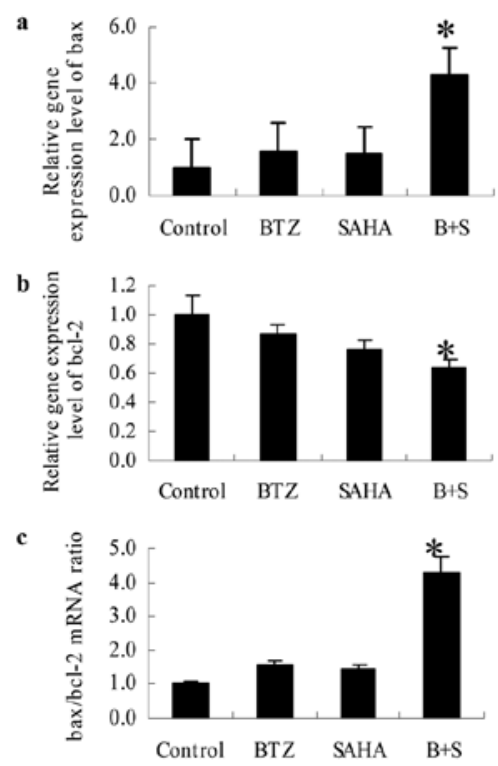

B

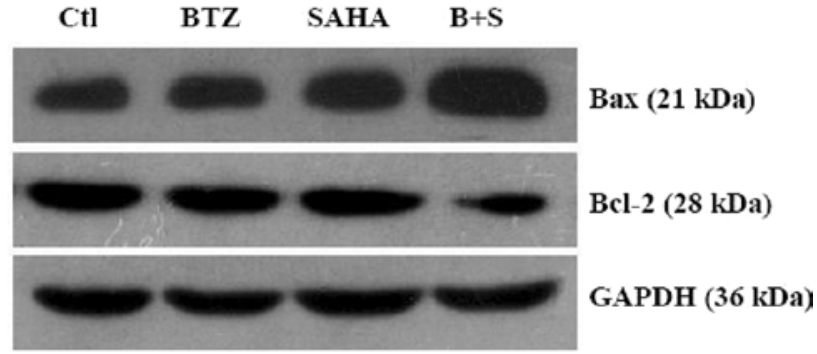

C

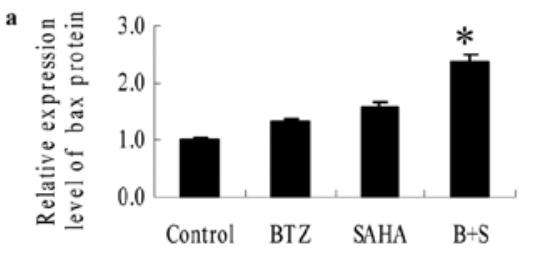

b

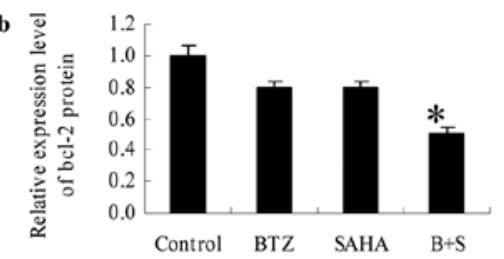

c

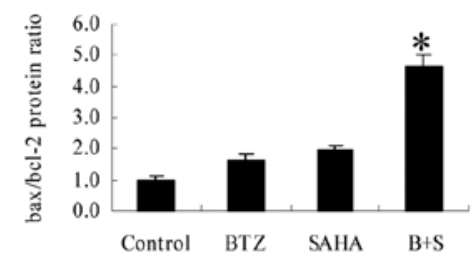

Figure 3. Increase in the expression ratio of bax/bcl-2 induced by the combination of bortezomib and SAHA. (A) Real-time PCR of the relative gene expression of bax, bcl-2 and the bax/bcl-2 ratio in HeLa cells after the indicated treatments. Gene expression levels were normalized to the level of $18 \mathrm{~S}$ rRNA expression. "P<0.05 vs. control. (B) Western blot analysis showing protein levels of bax, bcl-2 and GAPDH after the indicated treatments. (C) Relative protein expression of bax, bcl-2 and the bax/bcl-2 ratio after normalization to GAPDH. "P $<0.05$ vs. control. Data were obtained from at least three experiments.

A

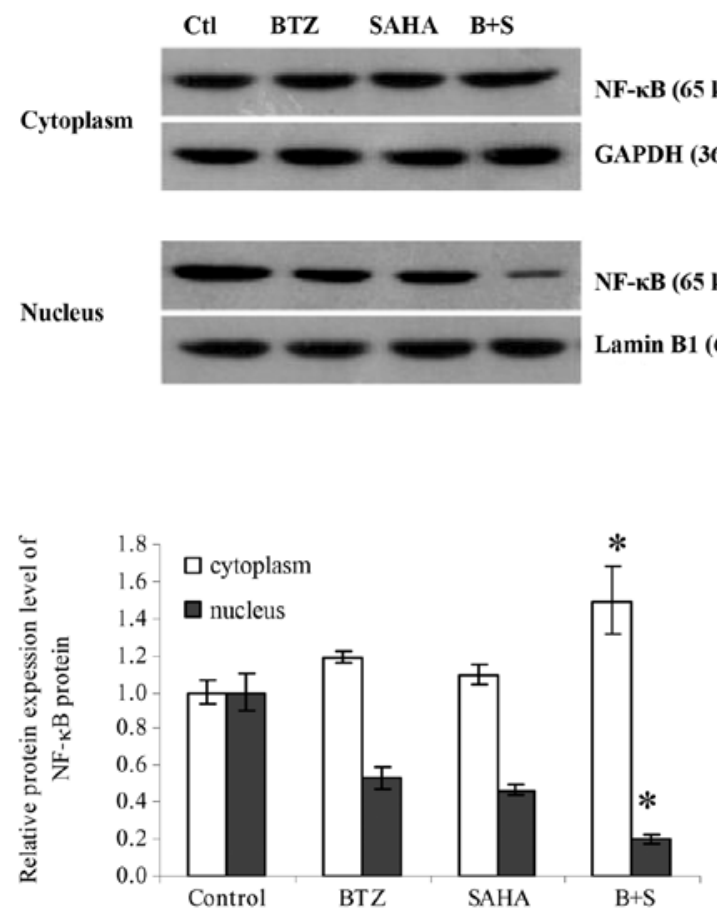

C
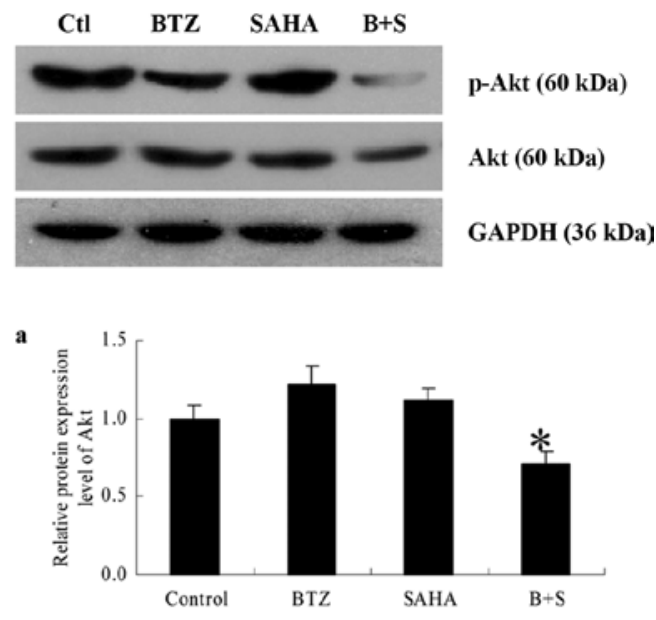

b

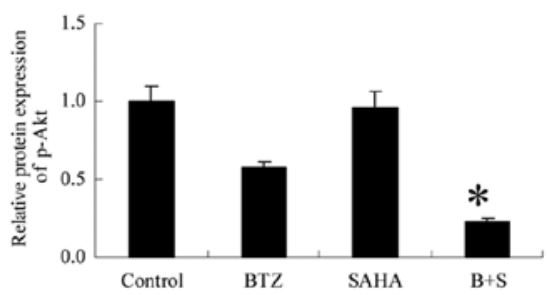

Figure 4. Effect of the combination of bortezomib and SAHA on the NF- $\mathrm{KB}$ and Akt signaling pathways. (A) Western blot analysis of the level of NF- $\mathrm{kB}$ in the nucleus and cytoplasm of HeLa cells after the indicated treatments. (B) Relative protein expression of cytoplasmic and nuclear NF- $\mathrm{KB}$ after normalization to GAPDH and Lamin B1. ${ }^{\text {P }}<0.05$ vs. control. (C) Western blot analysis of Akt and phosphorylated Akt (p-Akt) in HeLa cells after the indicated treatments. (D) Relative protein expression of p-Akt and Akt after normalization to GAPDH. "P<0.05 vs. control. Data were obtained from at least three experiments. 


\section{Discussion}

Human cervical carcinoma is a malignancy characterized by an aggressive course, a poor prognosis and a low chemosensitivity to common drugs. Bortezomib, a powerful proteasome inhibitor, targets the $26 \mathrm{~S}$ proteasome and leads to the induction of cellular apoptosis in various cancer cells (13). By promoting histone acetylation, chromatin uncoiling and the transcription of genes involved in multiple cellular processes, including apoptosis, SAHA exerts a marked apoptotic effect on a number of tumor cell types, whereas normal cells appear to be relatively resistant to the drug (14). Recently, these two agents have been evaluated in human cervical carcinoma and have been shown to have an anti-tumor effect in vitro and in vivo $(1,4,6)$. Our study shows that bortezomib and SAHA synergistically induce apoptosis in HeLa cells, associated with a marked increase in caspase-3 activation, the bax/bcl-2 ratio and the negative modulation of the NF- $\mathrm{KB}$ and Akt pathways.

The Bcl-2 protein family is known to constitute a central checkpoint during apoptosis. We studied the pro-apoptotic bcl-2 family member bax due to its central role in regulating cytochrome $c$ release in stressed cells. Overexpression of bax induces the release of cytochrome $c$ from mitochondria. Released cytochrome $c$ contributes to apoptosis by binding Apaf- 1 and caspase- 9 , whereas anti-apoptotic bcl-2 binds to bax and can form heterodimers, which block apoptosis in cells. The relative ratio of bax to bcl-2, rather than the single expression of either protein, prescribes survival or death to diverse apoptotic stimuli (15). Our data clearly demonstrate that co-treatment of HeLa cells with bortezomib and SAHA resulted in an increase in the level of bax with a concomitant decrease in bcl-2 levels and an increase in bax/bcl-2 ratio, as compared to cells treated with bortezomib or SAHA alone. The increase in the bax/bcl-2 ratio is concomitant with the morphological appearance of apoptosis and the activation of caspase- 3 , and may explain the synergistic mechanism of bortezomib and SAHA.

The NF- $\mathrm{KB}$ pathway is one of the most important signaling cascades, promoting the expression of proliferative and antiapoptotic genes. There is increasing evidence of the existance of alterations of the NF- $\mathrm{KB}$-associated pathways in HeLa cells (2). The blockade of IкBa degradation by proteasome inhibitors sequesters NF- $\kappa B$ into the cytosol, preventing its nuclear translocation and the activation of gene transcription (8). On the other hand, NF- $\mathrm{\kappa B}$ is regulated by reversible acetylation, therefore its activity may be modified by the action of HDAC inhibitors (16). HDAC inhibitors block the nuclear translocation of NF- $\mathrm{KB}$ via the acetylation of STAT1 (14). Taken together, the combination of bortezomib and SAHA may synergistically prevent NF- $\mathrm{KB}$ nuclear translocation and subsequent transactivation, which could lower the threshold for each drug's lethality. In our study, when bortezomib was used in combination with SAHA, a significant blockade of NF- $\mathrm{KB}$ nuclear translocation was observed, suggestive of their synergic mechanism as negative regulators of the NF- $\mathrm{KB}$ pathway.

The Akt pathway is another major anti-apoptotic and pro-survival pathway that is frequently hyperactivated in most human cancers. Activated p-Akt promotes cell growth and survival by inactivating downstream substrates such as Bad, procaspase-9 and Forkhead transcription factors (17). The proteasome inhibitor bortezomib is an agent that has multiple potential mechanisms of action, including the downregulation of Akt phosphorylation (18). In addition, SAHA may bind to HDAC, preventing the association of HDAC with specific phosphatases. These can then interact with Akt, inducing its dephosphorylation and inactivation (19). Our data show that the combination of bortezomib and SAHA attenuates the levels of the phosphorylated form of Akt and total Akt, indicating that Akt signaling further down-modulates the apoptotic threshold.

Both the NF- $\kappa \mathrm{B}$ and Akt cascades play a critical role in the resistance of cancer cells to chemotherapy and radiation therapy by protecting them from the induction of apoptosis (20). Previous studies have shown that Akt regulates the NF- $\mathrm{KB}$ pathway via the phosphorylation and activation of molecules in the NF- $\kappa B$ pathway (21). Furthermore, the disruption of the Akt pathway may contribute to the inactivation of NF- $\kappa B$, leading to a more lethal effect than the interruption of either pathway alone. Since NF- $\mathrm{KB}$ down-regulates the bax/bcl-2 ratio, its inactivation results in an increase of the bax $/ \mathrm{bcl}-2$ ratio and further caspase- 3 activation.

In conclusion, bortezomib interacts with SAHA by disrupting the Akt and NF- $\mathrm{kB}$ signaling pathways, thus triggering an increase in the bax/bcl-2 ratio and caspase-3 activation, eventually leading to the synergistic induction of apoptosis in HeLa cells. These findings indicate that this combination regimen is promising for the treatment of patients with human cevical carcinoma.

\section{References}

1. Monk BJ, Tewari KS and Koh WJ: Multimodality therapy for locally advanced cervical carcinoma: state of the art and future directions. J Clin Oncol 25: 2952-2965, 2007.

2. Aggarwal BB, Bhardwaj A, Aggarwal RS, Seeram NP, Shishodia S and Takada Y: Role of resveratrol in prevention and therapy of cancer: preclinical and clinical studies. Anticancer Res 24: 2783-2840, 2004.

3. Orlowski RZ and Dees EC: The role of the ubiquitinationproteasome pathway in breast cancer - applying drugs that affect the ubiquitin-proteasome pathway to the therapy of breast cancer. Breast Cancer Res 5: 1-7, 2003.

4. Birle DC and Hedley DW: Suppression of the hypoxia-inducible factor-1 response in cervical carcinoma xenografts by proteasome inhibitors. Cancer Res 67: 1735-1743, 2007.

5. Marks PA, Rifkind RA, Richon VM, Breslow R, Miller T and Kelly WK: Histone deacetylases and cancer: causes and therapies. Nat Rev Cancer 1: 194-202, 2001.

6. Andras IE, Rha G, Huang W, et al: Simvastatin protects against amyloid beta and HIV-1 tat-induced promoter activities of inflammatory genes in brain endothelial cells. Mol Pharmacol 73: 1424-1433, 2008.

7. Zhang QL, Wang L, Zhang YW, et al: The proteasome inhibitor bortezomib interacts synergistically with the histone deacetylase inhibitor suberoylanilide hydroxamic acid to induce T-leukemia/ lymphoma cells apoptosis. Leukemia 23: 1507-1514, 2009.

8. Heider U, von Metzler I, Kaiser M, et al: Synergistic interaction of the histone deacetylase inhibitor SAHA with the proteasome inhibitor bortezomib in mantle cell lymphoma. Eur J Haematol 80: 133-142, 2008

9. Servida F, Soligo D, Delia D, et al: Sensitivity of human multiple myelomas and myeloid leukemias to the proteasome inhibitor I. Leukemia 19: 2324-2331, 2005.

10. Lee JJ, Kong M, Ayers GD and Lotan R: Interaction index and different methods for determining drug interaction in combination therapy. J Biopharm Stat 17: 461-480, 2007.

11. Guerra S, Lopez-Fernandez LA, Pascual-Montano A, Najera JL, Zaballos A and Esteban M: Host response to the attenuated poxvirus vector NYVAC: upregulation of apoptotic genes and NF-kappa B-responsive genes in infected HeLa cells. J Virol 80: 985-998, 2006. 
12. Bhushan S, Malik F, Kumar A, et al: Activation of p53/p21/ PUMA alliance and disruption of PI-3/Akt in multimodal targeting of apoptotic signaling cascades in cervical cancer cells by a pentacyclic triterpenediol from boswellia serrata. Mol Carcinog 48: 1093-1108, 2009.

13. Nalepa G and Harper JW: Therapeutic anti-cancer targets upstream of the proteasome. Cancer Treat Rev 29: 49-57, 2002.

14. Buchwald M, Kramer OH and Heinzel T: HDACi - targets beyond chromatin. Cancer Lett 280: 160-167, 2009.

15. Wang J, Wu AB, Xu YF, Liu JW and Qian XH: M-2-A induces apoptosis and $\mathrm{G}(2)-\mathrm{M}$ arrest via inhibiting PI3 K/Akt pathway in HL60 cells. Cancer Lett 283: 193-202, 2009.

16. Mayo MW, Denlinger CE, Broad RM, et al: Ineffectiveness of histone deacetylase inhibitors to induce apoptosis involves the transcriptional activation of NF-kappa B through the Akt pathway. J Biol Chem 278: 18980-18989, 2003.

17. Gao X, Deeb D, Jiang H, Liu Y, Dulchavsky SA and Gautam SC: Synthetic triterpenoids inhibit growth and induce apoptosis in human glioblastoma and neuroblastoma cells through inhibition of prosurvival Akt, NF-kappa B and Notch1 signaling. J Neurooncol 84: 147-157, 2007.
18. Yu CR, Friday BB, Lai JP, et al: Cytotoxic synergy between the multikinase inhibitor sorafenib and the proteasome inhibitor bortezomib in vitro: induction of apoptosis through Akt and c-Jun NH2-terminal kinase pathways. Mol Cancer Ther 5: 2378-2387, 2006

19. Emanuele S, Lauricella M, Carlisi D, et al: SAHA induces apoptosis in hepatoma cells and synergistically interacts with the proteasome inhibitor bortezomib. Apoptosis 12: 1327-1338, 2007.

20. Maddika S, Ande SR, Panigrahi S, et al: Cell survival, cell death and cell cycle pathways are interconnected: implications for cancer therapy. Drug Resistance Updates 10: 13-29, 2007.

21. Sarkar $\mathrm{FH}$ and $\mathrm{Li}$ YW: Cell signaling pathways altered by natural chemopreventive agents. Mut Res 555: 53-64, 2004. 